\title{
FAST REACTOR MULTIPHYSICS AND UNCERTAINTY PROPAGATION WITHIN WIMS
}

\author{
Ben Lindley ${ }^{1}$, Brendan Tollit ${ }^{1}$, Peter Smith ${ }^{1}$, Alan Charles ${ }^{1}$, Robert Mason ${ }^{1}$, Tim Ware ${ }^{1}$, \\ Ray Perry $^{1}$, Jean Lavarenne ${ }^{1}$, Una Davies ${ }^{2}$, Robert Gregg ${ }^{3}$ \\ ${ }^{1}$ Wood \\ Kings Point House, Queen Mother Square, Poundbury, Dorchester, DT1 3BW, UK \\ ${ }^{2}$ University of Cambridge \\ Cambridge University Engineering Department, Cambridge, CB1 1PZ, UK \\ ${ }^{3}$ National Nuclear Laboratory \\ Preston Laboratory, Preston, PR4 0XJ, UK
}

ben.lindley@woodplc.com, brendan.tollit@woodplc.com, peter.smith2@woodplc.com, alan.charles@woodplc.com, robert.mason@woodplc.com, tim.ware@woodplc.com, ray.perry@,woodplc.com, ud215@,cam.ac.uk, robert.wh.gregg@uknnl.com

\begin{abstract}
For liquid metal-cooled fast reactors (LMFRs), improved predictive modelling is desirable to facilitate reactor licensing and operation and move towards a best estimate plus uncertainty (BEPU) approach. A key source of uncertainty in fast reactor calculations arises from the underlying nuclear data. Addressing the propagation of such uncertainties through multiphysics calculations schemes is therefore of importance, and is being addressed through international projects such as the Sodium-cooled Fast Reactor Uncertainty Analysis in Modelling (SFR-UAM) benchmark. In this paper, a methodology for propagation of nuclear data uncertainties within WIMS is presented. Uncertainties on key reactor physics parameters are calculated for selected SFR-UAM benchmark exercises, with good agreement with previous results. A methodology for coupled neutronic-thermal-hydraulic calculations within WIMS is developed, where thermal feedback is introduced to the neutronic solution through coupling with the ARTHUR subchannel code within WIMS and applied to steady-state analysis of the Horizon 2020 ESFR-SMART project reference core. Finally, integration of reactor physics and fuel performance calculations is demonstrated through linking of the WIMS reactor physics code to the TRAFIC fast reactor fuel performance code, through a Fortran-C-Python (FCP) interface. Given the 3D multiphysics calculation methodology, thermal-hydraulic and fuel performance uncertainties can ultimately be sampled alongside the nuclear data uncertainties. Together, these developments are therefore an important step towards enabling propagation of uncertainties through high fidelity, multiphysics SFR calculations and hence facilitate BEPU methodologies.
\end{abstract}

KEYWORDS: WIMS, TRAFIC, SFR-UAM, ESFR-SMART 


\section{INTRODUCTION}

For liquid metal-cooled fast reactors (LMFRs), improved predictive modelling is desirable to facilitate reactor licensing and operation and move towards a best estimate plus uncertainty (BEPU) approach. This is particularly important for innovative reactor designs (e.g. axially heterogeneous cores) for which experimental data may be more limited. A key source of uncertainty in fast reactor calculations arises from the underlying nuclear data. Addressing the propagation of such uncertainties through multiphysics calculations schemes is therefore of importance, and is being addressed through international projects such as the Sodium-cooled Fast Reactor Uncertainty Analysis in Modelling (SFR-UAM) benchmark [1].

Developed by the ANSWERS ${ }^{\circledR}$ Software Service in Wood, the WIMS reactor physics code has been developed, benchmarked and validated for reactor physics analysis of LMFRs [2] [3]. This paper presents developments towards extending this capability for multiphysics analysis of fast reactors under uncertainty. Three elements are presented in this paper, which in combination ultimately provide this capability:

- Development of an uncertainty analysis methodology. This is based on sampling of input parameters based on their uncertainties. The particular focus of this study is on nuclear data uncertainty propagation, as this presents particular challenges. Here, the developed methodology is applied to the SFR-UAM benchmark. It is envisaged that such sampling of nuclear data can be combined with sampling of other parameters (e.g. engineering tolerances). To this end, the ANSWERS ${ }^{\circledR}$ Visual Workshop GUI has been designed to generate and run a large number of input files with stochastically-varying input parameters. It can also be used to automate the execution of calculations with the sampled nuclear data libraries [4].

- Development of a coupled neutronic-thermal-hydraulic calculation scheme. While it is typical to perform 3D neutronic calculations for fast reactors, 3D coupled calculations are becoming more common, and in particular are being addressed within the current ESFR-SMART Horizon 2020 project [5]. Recently, coupled calculation schemes have been developed in PARCS-TRACE [6], OpenFOAM [6] and DYN3D [7]. A particular challenge of coupled fast reactor modelling is treatment of differential thermal expansion of the fuel assemblies. A capability to model this has now been added to WIMS, and demonstration coupled neutronic-thermal-hydraulic calculations are performed for the ESFR-SMART core.

- Development of a link between WIMS and the TRAFIC fast reactor fuel performance code [8]. A one-way linking is under development between WIMS and TRAFIC, where a single representative pin per fuel assembly is modelled in TRAFIC, utilizing time-dependent data from WIMS (including axial power and flux profile). Further developments of this capability are targeted within the UK BEIS national R\&D programme in reactor physics.

\section{NUCLEAR DATA UNCERTAINTY PROPAGATION}

\subsection{Introduction}

The OECD/NEA SFR-UAM benchmark targets code-to-code comparison of neutronic reactivity coefficients and associated uncertainties, and their impact on transient analysis [1]. Two cores are considered: a large oxide core and a medium metallic core - the latter is considered in the present work and is based on the 1000 MWth Advanced Burner Reactor concept proposed by ANL. Results for several participants were reported in Ref [1] in 2017. Since then, the benchmark has been progressed (and the solutions discussed in this section have also been submitted to the benchmark). 


\subsection{Calculation Methodology}

The WIMS fast reactor neutronics calculation methodology has been described previously, for example in Ref. [3]. Briefly, a 1968-group slowing down calculation is performed using the WIMSECCO module within WIMS (an implementation of the ECCO cell code [9]) to generate 172-group cross sections. The SPH method is used for homogenization of control assemblies. The SP3 method in RZ geometry is used to condense to 33 groups, and an SP3 calculation in hexagonal-Z geometry using the MERLIN module within WIMS is used for the core flux solution. Calculations are performed using the JEFF-3.1.2 nuclear data library.

The present work considers development of a scheme for nuclear data uncertainty analysis. A sampling methodology is used, where perturbations are calculated from a covariance library using Latin Hypercube Sampling. The ANSWERS ${ }^{\circledR}$ codes utilize a 44 group covariance library compiled from the best available data from multiple data libraries, as described in Ref. [4]. Cross-section perturbations are applied to the reconstructed point energy (PENDF) file produced using NJOY [10]. Sampled ENDF and PENDF files are used to generate perturbed NJOY data for cross sections, nu-bar and fission spectrum. Sampled 172 group nuclear data libraries have been produced in this manner for use in WIMS for thermal reactor applications. Here, this process is extended to the 1968 group libraries used in the WIMS fast reactor resonance shielding treatment. In particular, the CALENDF code [11] is used to generate cross-sections in the resonance range, including probability table (PT) data to represent resonance shielding. For generating sampled libraries, scaling factors are applied to the cross-sections output from CALENDF, consistent with those used to produce the sampled PENDF files. CALENDF and NJOY data is then combined to form sampled data for the ECCO library (Figure 1).

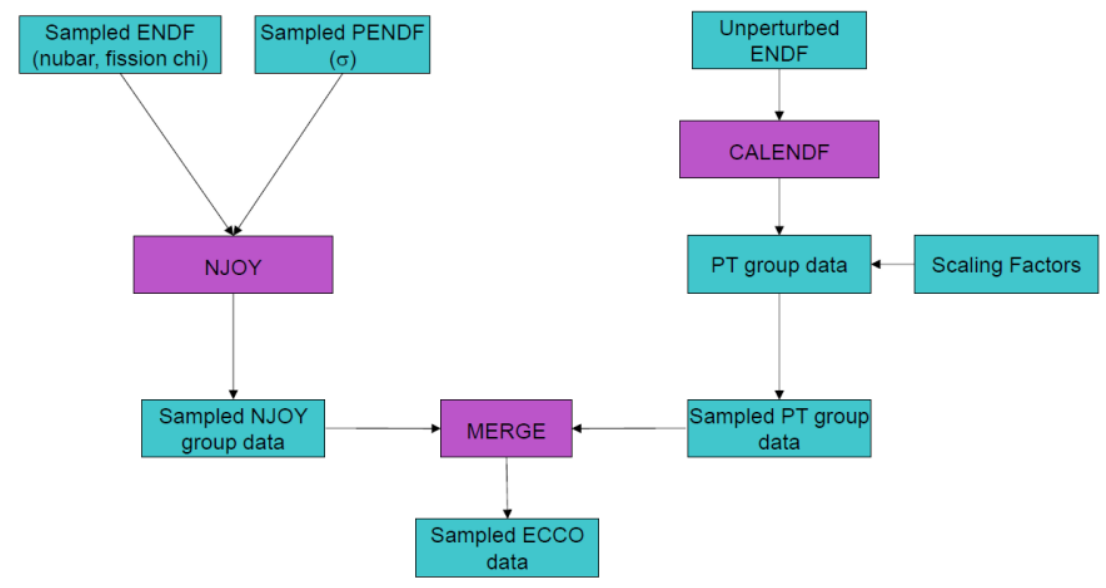

Figure 1. WIMS fast reactor perturbed nuclear data library generation process

A sample of 25 perturbed nuclear data libraries (sufficient for a $90 \% / 90 \%$ tolerance interval) is generated for the present work in order to perform proof of concept, subsequent to which it is straightforward to scale up the number of libraries. A rough estimate for the statistical error on the uncertainty can be performed. Under the assumption of a normal distribution of $n$ sampled outputs of standard deviation $s d$, the standard error on the standard deviation (sesd) is:

$$
\text { sesd }=s d / 2 \sqrt{n}
$$

For 25 sampled libraries, this is $10 \%$. For nuclear data uncertainties, this is sufficiently low for proof of concept and high level comparison with other participants. The sampled libraries could be combined with a Latin hypercube sample of the engineering tolerances (with the same sample size) to treat both simultaneously, i.e. to sample all uncertain variables within the same Latin hypercube sample. 


\subsection{Results}

Best estimate results nuclear data uncertainties for WIMS are presented in Table I, compared to the average reported by participants in the 2017 paper [1]. WIMS best estimates are essentially within $1 s d$ of the reported mean (across six participants) except the control rod worth. Here, the discrepancy between WIMS and reported continuous energy Monte Carlo (MC) solutions is comparable with that between reported deterministic and reported MC solutions, but with opposite sign, so results are reasonable. Such discrepancies are likely to arise from the implementation of the SPH correction, but improvement of best estimate neutronics calculations is the scope of this paper.

Table I.: WIMS results for SFR-UAM Medium Metallic Core compared to benchmark average reproduced from Ref. [1]

\begin{tabular}{|c|c|c|c|c|c|}
\hline Parameter & WIMS & $\begin{array}{l}\text { Benchmark } \\
\text { Av [1] }\end{array}$ & $\begin{array}{l}\text { Benchmark } \\
\text { SD [1] }\end{array}$ & $\begin{array}{l}\text { WIMS } \\
\text { Uncertainty }\end{array}$ & $\begin{array}{l}\text { Benchmark } \\
\text { Uncertainty } \\
\text { Range [1] }\end{array}$ \\
\hline Keff & 1.02387 & 1.0216 & 0.0066 & $1.0 \%$ & $1.3-1.5 \%$ \\
\hline $1 \% \mathrm{Na}(\mathrm{pcm} / \mathrm{K})$ & 0.41 & 0.380 & 0.028 & $10.2 \%$ & $13.8-18.1 \%$ \\
\hline $1 \%$ Wrapper $(\mathrm{pcm} / \mathrm{K})$ & 0.02 & 0.022 & 0.001 & $5.4 \%$ & \\
\hline $1 \%$ Clad $(\mathrm{pcm} / \mathrm{K})$ & 0.05 & 0.046 & 0.004 & $5.1 \%$ & \\
\hline $1 \%$ Fuel $(\mathrm{pcm} / \mathrm{K})$ & -0.56 & -0.565 & 0.019 & $0.9 \%$ & \\
\hline 1\% Fuel + Axial (pcm/K) & -0.26 & -0.272 & 0.018 & $1.7 \%$ & $2.4-2.7 \%$ \\
\hline $1 \%$ Grid $(\mathrm{pcm} / \mathrm{K})$ & -1.09 & -1.113 & 0.032 & $1.1 \%$ & $1.9-2.8 \%$ \\
\hline CR Worth (pcm) & -9318 & -9742 & 202 & $2.0 \%$ & $2.5-2.8 \%$ \\
\hline $\begin{array}{l}\text { CR Worth } \\
(5 \mathrm{~cm} \text { inserted) (pcm) }\end{array}$ & -232 & -236 & 5 & $2.3 \%$ & \\
\hline DNF (pcm) & 345 & 339 & 10 & $1.2 \%$ & $1.1 \%$ \\
\hline Doppler const (pcm) & -379 & -383 & 19 & $3.7 \%$ & $5.8-7.5 \%$ \\
\hline Na Void Worth (pcm) & 1485 & 1361 & 131 & $14.1 \%$ & $19.2-28.2 \%$ \\
\hline
\end{tabular}

WIMS results for nuclear data uncertainty are compared to results in Ref [1] for two other participants. WIMS uncertainties are generally lower than those reported previously, potentially due to use of a different covariance matrix. As more results become available through the benchmark activities, further comparison can be performed.

\section{COUPLED NEUTRONIC-THERMAL-HYDRAULIC CORE ANALYSIS}

\subsection{Introduction}

The ESFR-SMART Horizon 2020 project aims at enhancing the safety of SFRs, in particular the commercial size ESFR concept [5]. To this end, a reference 3600 MWth core is defined with 504 fuel assemblies, inner and outer fuel zoning, lower axial blanket and upper sodium plenum. The conversion ratio of the core is around unity. Within ESFR-SMART, simulations with coupled spatial neutronics and thermalhydraulics codes are being performed to provide a realistic temperature distribution within the core, using parameterized multigroup cross sections. 


\subsection{Calculation Methodology}

The WIMS neutronic calculation methodology is as described in Section 2. The ARTHUR subchannel thermal-hydraulics module within WIMS [12] is then used to solve for the coolant and fuel temperature distribution, with the core modelled with one coolant channel per assembly. The mass, momentum and energy equations are solved for the coolant temperature distribution in each channel, and the conduction equation is solved radially for a representative pin in each assembly for each axial slice. The heat transfer coefficient between the coolant and the clad outer surface uses the correlation in Ref. [13]. The gap conductance is parameterized as a function of linear heat rate and irradiation as described in Ref. [14].

MERLIN and ARTHUR are coupled together through the CAMELOT framework within WIMS. The thermal feedback model utilizes parameterized cross sections for fuel temperature (including reduction in density) and sodium voiding. The variation in fuel temperature across the reactor leads to a thermal expansion effect where the assemblies are all of different heights, which presents difficulties when using a structured mesh (as utilized here). Using a similar approach to Ref. [7], this is handled through smearing the composition of the fuel and plenum using appropriate volume fractions, for the axial layers at the tops of each fuel assembly. This gives a consistent treatment of the variation of axial expansion across the core. Here it is assumed that the fuel is not mechanically linked to the clad, and hence expands in isolation to the clad. However it is noted that under irradiation, it is possible for the fuel to become mechanically linked to the clad, which would reduce the expansion effect.

\subsection{Results}

The power distribution at EOEC in the ESFR-SMART core is first calculated without thermal feedback (shown in Figure 2), and is in excellent agreement with results from the continuous energy Monte Carlo code Serpent [15] with an rms discrepancy of $0.7 \%$, and a maximum discrepancy (in the peripheral fuel elements of $2.1 \%$ ). k-eff from WIMS also agrees to within $\sim 200 \mathrm{pcm}$ of the Serpent value.

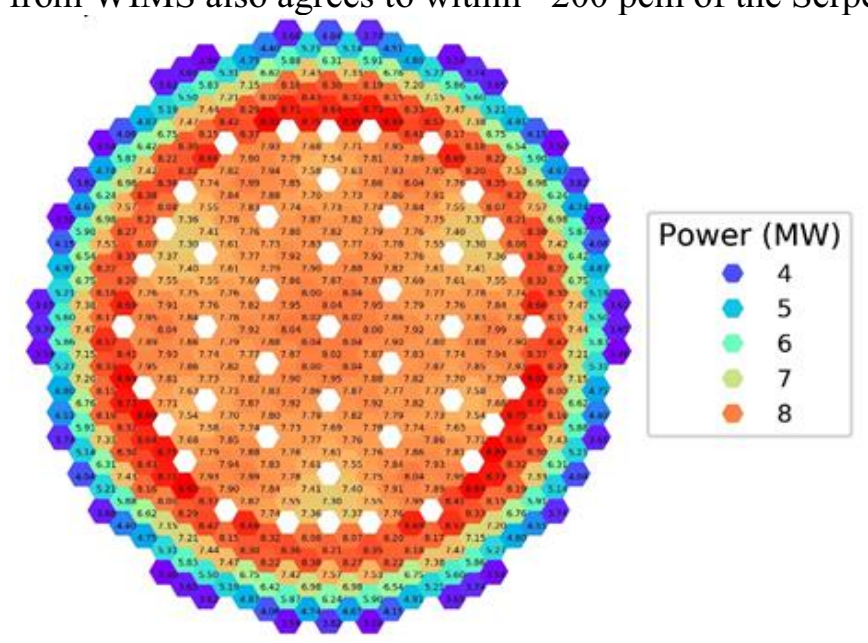

Figure 2. Power Distribution (MW) at EOEC for ESFR-SMART core, calculated using WIMS without thermal feedback

A coupled calculation is then performed with thermal-hydraulic feedback. At time of writing, the calculation route and related task within the ESFR-SMART project are ongoing. Presented calculations are therefore work in progress. The \% variation between calculations with and without thermal feedback is given in Figure 3. The effect of thermal feedback on the power is predicted to be up to $\sim 3 \%$. The axially integrated fuel and coolant temperature distributions are given in Figure 4. In future work, these results will be 
compared with calculations by other organizations within the framework of the ESFR-SMART project. The impact of thermal feedback on the Doppler coefficient and sodium void worth will also be calculated.

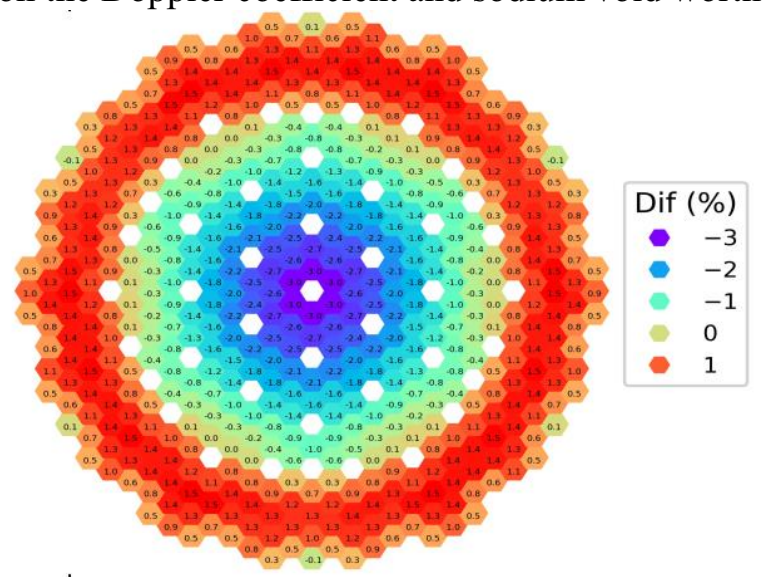

Figure 3. Percentage variation in power distribution at EOEC for ESFR-SMART core, calculated with and without thermal feedback
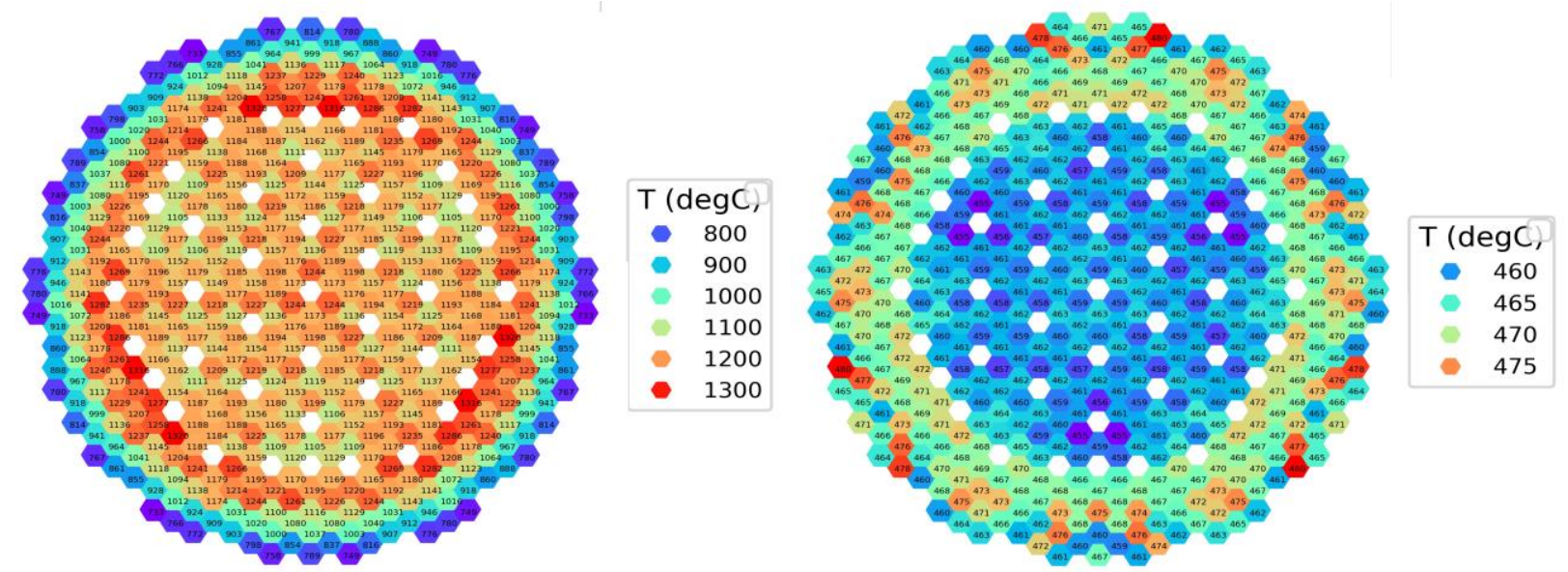

Figure 4. Fuel (left) and coolant (right) temperature distribution (K) at EOEC for ESFR-SMART core, calculated using WIMS with thermal feedback

\section{INTEGRATION OF FUEL PERFORMANCE MODELLING}

A coupling has been developed between WIMS and the TRAFIC fuel performance code. TRAFIC is a mechanistic, 1.5-dimensional fast reactor fuel performance code developed by Wood. There are variants for oxide, carbide and nitride fuels, which have a shared code base. The validation of TRAFIC relies on comparison with data from post-irradiation examination and results from various transient tests.

To facilitate multiphysics coupling in a general sense, a Fortran-C-Python interface has been implemented within WIMS, which allows WIMS to call an external python script; passing the script interface data and allowing the script to alter this data. While TRAFIC does not presently have functionality for in-memory coupling, WIMS-TRAFIC calculation can hence be implemented by using the python script as an adapter to write the TRAFIC input file and, if two-way coupling is desired, read the corresponding output file.

A one-way coupling has been implemented, where the axial power distribution from WIMS is utilized within a representative TRAFIC simulation for each fuel assembly. In this manner, the actual core 3D power solution is utilized within the fuel performance simulations, improving the accuracy to which fuel performance can be predicted. The capability is being developed to extend this to a two-way coupling, if 
desired by the user, where fuel and clad temperatures and axial swelling are calculated by TRAFIC and fed back into WIMS.

Further work, notably within the UK national R\&D programme in reactor physics, will utilize this capability to perform a demonstration case where a TRAFIC simulation will be run for a representative pin within each assembly of a fast reactor core, with data being provided by WIMS on a time-dependent basis:

- Axial power shape

- Axial variation of outer clad temperature calculated as described in Section 3

- Axial variation in clad damage (in dpa NPRT).

\section{CONCLUSIONS}

In combination, the developments presented in this paper represent substantial progress towards a multiphysics fast reactor core calculation scheme (Figure 5). Given the 3D multiphysics calculation methodology, thermal-hydraulic and fuel performance uncertainties can ultimately be sampled alongside the nuclear data uncertainties. Together, these developments are therefore an important step towards enabling propagation of uncertainties through high fidelity, multiphysics SFR calculations and hence facilitate BEPU methodologies.

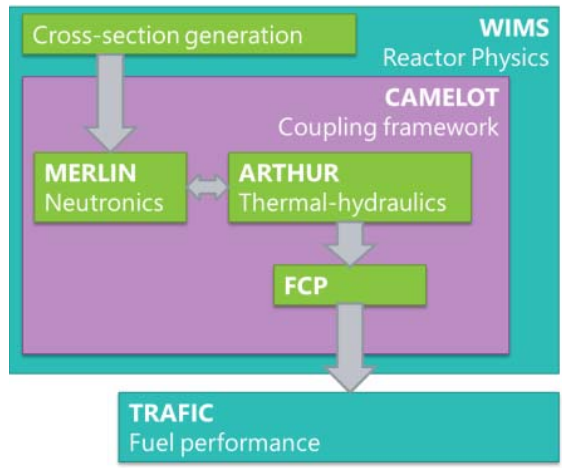

Figure 5. WIMS fast reactor calculation scheme, under development

Previous recent developments are:

- Whole core fast reactor neutronics calculation capability within WIMS

- Development of a subchannel thermal hydraulic solver within WIMS

- Development of a tool for sampling and uncertainty quantification (UQ) within the Visual Workshop GUI

- Methodology for nuclear data UQ for thermal reactors.

New developments summarized in this paper are:

- Extension of the nuclear data UQ methodology to fast reactors, with application to the SFR-UAM benchmark

- Implementation of neutronic-thermal-hydraulic coupled calculation scheme for fast reactors, including treatment of axial thermal expansion. This is being applied to the ESFRSMART core. Preliminary results are presented, with work ongoing.

- Implementation of a WIMS-TRAFIC reactor physics + fuel performance coupling mechanism using a Fortran-C-Python interface. 
Further work will focus on integrating these components of the calculation sequence, potentially through (1) extending work on uncertainty analysis to consider coupled neutronic-thermalhydraulic steady-state and transient modelling (2) demonstrating the reactor-physics + fuel performance link, for neutronic and/or neutronic-thermal-hydraulic cases.

\section{ACKNOWLEDGMENTS}

This paper contains work prepared within the EU Project ESFR-SMART which has received funding from the EURATOM Research and Training Programme 2014-2018 under Grant Agreement No. 754501.

\section{REFERENCES}

[1] N. E. Stauff et al., "Evaluation of the OECD/NEA/SFR-UAM Neutronics Reactivity Feedback and Uncertainty Benchmarks," in International Conference on Fast Reactors and Related Fuel Cycles (FR17), Yekaterinburg, Russia, 2017.

[2] B. Lindley et al., "Developments with the WIMS Reactor Physics Code for Whole Core Calculations," in $M \& C$ 2017, Jeju, Korea, April 16-20 2017.

[3] U. Davies, B. Lindley, B. Tollit and E. Shwageraus, "Whole-core validation of the Superphenix reactor using WIMS11 and an investigation into a hybrid RZ-HEX SP3 calculation route," in M\&C2019, Portland, OR, USA, 2019.

[4] J. G. Hosking et al., "Development of a reactor physics uncertainty quantification toolkit for use with the WIMS and MONK codes," in PHYSOR 2018, Cancun, Mexico, 2018.

[5] K. Mikityuk et al., "ESFR-SMART: new Horizon 2020 project on SFR safety," in International Conference on Fast Reactors and Related Fuel Cycles (FR17), Yekaterinburg, 2017.

[6] C. Fiorina, I. Clifford, M. Aufiero and K. Mikityuk, "GeN-Foam: A novel OpenFOAM(R) based multi-physics solver for 2D/3D transient analysis of nuclear reactors," Nuclear Engineering and Design, vol. 294, pp. 24-37, 2015.

[7] E. F. E. Nikitin, "Extension of the reactor dynamics code DYN3D to SFR applications - Part I: Thermal expansion models," Annals of Nuclear Energy, vol. 119, pp. 382-389, 2018.

[8] R. Calabrese, F. Vettraino, C. Artioli, V. Sobolev and R. Thetford, "Heterogeneous fuels for minor actinides transmutation: Fuel performance codes predictions in the EFIT case study," Annals of Nuclear Energy, vol. 37 6, pp. 867-874, 2010.

[9] G. Rimpault et al., "The ERANOS code and data system for fast reactor neutronic analysis," in PHYSOR2002, Seoul, Korea, Oct 7-10, 2002.

[10] R. E. MacFarlane et al., "The NJOY Nuclear Data Processing System Version 2012. LA-UR-1227079," Los Alamos National Laboratory, 2012.

[11] J. C. Sublet et al., "CALENDF-2010: User Manual. CEA-R-6277," 2011.

[12] B. Tollit et al., "Development of a subchannel model within the ANSWERS Software Service WIMS reactor physics code," in PHYSOR 2018, Cancun, Mexico, Apr 22-26 2018.

[13] K. Mikityuk, "Heat transfer to liquid metal: Review of data and correlations for tube bundles," Nuclear Engineering and Design, vol. 239, no. 4, pp. 680-7, 2009.

[14] J. Lavarenne et al., "A 2-D correlation to evaluate fuel-cladding gap thermal-conductance in mixed oxide fuel elements for sodium-cooled fast reactors," in GLOBAL 2019, Seattle, USA, 2019.

[15] E. Fridman, "Current applications of Serpent in the Euratom project ESFR-SMART," in 8th International Serpent User Group Meeting, Espoo, Finland, 2018. 